\title{
Nuclear level density and related physics
}

\author{
Vladimir Zelevinsky ${ }^{1, *}$ and Sofia Karampagia ${ }^{2, * *}$ \\ ${ }^{1}$ Department of Physics and Astronomy and National Superconducting Cyclotron Laboratory/Facility for Rare Isotope Beams, \\ Michigan State University, East Lansing, Michigan 48824-1321 USA \\ ${ }^{2}$ Department of Physics, Grand Valley State University, Allendale, Michigan 49504 USA
}

\begin{abstract}
The knowledge of the level density as a function of excitation energy and nuclear spin is necessary for the description of nuclear reactions and in many applied areas. We discuss the level density problem as a part of the general understanding of mesoscopic systems with strong interactions. The underlying physics is that of quantum chaos and thermalization which allows one to use statistical methods avoiding full diagonalization. The resulting level density is well described by the constant temperature model in agreement with experimental data. We discuss the effective temperature parameter and show that it is not related to the pairing phase transition being analogous to the limiting temperature in particle physics. Other aspects of underlying physics include the collective enhancement of the level density, random coupling of individual spins and the role of incoherent collision-like interactions.
\end{abstract}

\section{Introduction}

The probabilities of nuclear reactions, including those in atomic industry and in astrophysical conditions, require for their prediction the knowledge of the level density of final nuclei. At energies near the ground state, the levels often can be directly measured. At higher excitation energy, as a rule, there are quite dense sequences of the states with the same exact quantum numbers. In special cases, as reactions induced by slow neutrons of known energy, the individual states can be resolved; in fact here we deal with resonances but in the situation where they do not overlap and can be counted individually.

As discussed in more detail later, with excitation energy growing, the dynamical properties of neighboring states tend to become quite similar due to the strong mixing of simple particle-hole configurations by the nucleon interactions. This mixing is getting really strong because of the combinatorial growth of the number of possible elementary excitations that unavoidably leads to a dense sequence of levels. The states with the same total spin $J$, its projection $M$, isospin and parity (in a good approximation), starting from a certain excitation energy form a "disordered crystal" [1] with statistical properties locally close to the limit of the Gaussian Orthogonal Ensemble (GOE) which parameters smoothly change along the energy scale. But the random matrix theory does not help in finding the global level density $\rho(E)$ as a function of excitation energy.

To predict precisely the level density of a complex quantum system, one needs to solve the many-body problem. As this is possible only in very light systems or

\footnotetext{
*e-mail: zelevins@nscl.msu.edu

**e-mail: karampso@gvsu.edu
}

in oversimplified models, the physical approximations are needed. The two main directions are the mean-field methods and configuration interaction approaches. The modern mean-field theory, originated in the Fermi-gas model, is based on the particle-hole combinatorics and includes in some form the pairing interaction [2]. The configuration interaction methods use the matrix diagonalization [3] in a physically truncated orbital space (the shell-models with the currently achievable dimension of the order $10^{11}$ ), the schemes based on the Monte Carlo procedures or statistical spectroscopy. We will argue that the statistical moments method [4] is essentially equivalent to full diagonalization giving practically exact answers for given space. Both these approaches lose their validity at energy where the states outside the working space appear as intruders. The Monte Carlo techniques [5, 6], as well as mean-field methods, account only for the most regular part of interparticle interactions, while they do not include incoherent collison-like processes which are equally important in forming the realistic level density.

The shell-model calculations taking into account all parts of the interactions show that the typical nuclear level density behaves as in the so-called constant temperature model (CTM) for energies up to $12-15 \mathrm{MeV}$ (sometimes even higher) which well describes experimental data. Here $\rho(E) \propto \exp (E / T)$ with a constant effective temperature parameter $T$. Below we show the level density resulting from the shell model, the quality of its description at low energy by the CTM and the evolution of the effective temperature along the nuclear chart. The phenomenologically known collective enhancement of the level density will be illustrated by the change of the level density under the nuclear shape transitions. We conclude with the discussion of the meaning of the CTM arguing that the frequent interpreta- 
tion [7] in terms of the phase transition from the superfluid to normal phase at fixed temperature is not supported by the shell model data.

\section{Shell-model level density}

The advanced versions of the shell model are defined by a set of single-particle orbitals and matrix elements of all (usually only pairwise) nucleon interactions allowed by the conservation laws. These quantities are determined by comparison of the derived spectroscopic information with the available data. We know that many matrix elements are not uniquely defined by the experiments. However, their presence is important for the correct level density. It is also clear that the shell-model Hamiltonian is not given by a random matrix of uncorrelated elements. Indeed, any twobody process with fixed initial and final orbitals can happen on a different background of other occupancies and therefore the same matrix element repeatedly appears in the Hamiltonian matrix.

In spite of that, the resulting energy spectrum is similar in many respects to the spectra of random matrix ensembles [3]. The natural mechanism of this chaotization can be traced through the process of switching on the interparticle interactions. The eigenstates with the same exact quantum numbers travel through the sequence of multiple avoided crossings coming out at the realistic interaction strength as extremely complicated superpositions of many simple particle-hole configurations. Their mutual repulsion creates the spectral sequences similar to those in the GOE. We come to what can be called quantum chaos. The global level density is typically given by the Gaussian curve with the centroid maximum at energy $E_{m}$, the width $\sigma$, and particle-hole symmetry. Although only the left low part corresponds to realistic physics, the whole curve is instructive as an example of the exact solution of the quantum many-body problem.

Along the level density curve, all observables are expressed as almost unique functions of energy, so that they can be reinterpreted as thermodynamic variables, in spite of absence of an external thermostat. The corresponding thermodynamic temperature is expressed as

$$
T_{\text {global }}=\frac{\sigma^{2}}{E_{m}-E} ;
$$

it is positive on the left branch, jumps from $+\infty$ to $-\infty$ at the center and becomes negative on the right branch. All observables are smoothly changing along the spectrum as a consequence of the chaotic mixing. This global behavior is in extremely good agreement with the fermionic statistics. Namely, we can find, for each individual eigenstate, occupation numbers of single-particle orbitals. They are well fit by a Fermi-function with certain parameters of temperature and chemical potential. The values of temperature found in this way are in perfect agreement with the global temperature (1).

The tails of the Gaussian expression (1), where the chaoticity is not complete, are not fully appropriate at very low (and very high) excitation energy. As many states of experimental interest are located here, one needs a special treatment. Phenomenologically, one can use the backshifted Fermi gas formula [8], where the main energy dependence $\propto \exp (\sqrt{2 a E})$ is defined by the fitted level density parameter $a$. Its value typically is to be taken greater than it would be from the single-particle level density at the Fermi surface; this reflects a stronger energy dependence, compare with CTM, Section 4.

\section{Moments method}

Instead of the diagonalization of extremely large matrices, one can successfully use the ideas of statistical spectroscopy [4, 9] exploiting just the lowest moments of the Hamiltonian. The orbital space is divided into partitions $p$, allowed configurations of particles with certain exact characteristics $\alpha$ and dimensions $D_{\alpha p}$. The mean energy of a partition is defined by the trace

$$
E_{\alpha p}=\overline{H_{\alpha p}} \equiv \frac{1}{D_{\alpha p}} \operatorname{Tr}^{(\alpha p)} H .
$$

These centroids form a smooth sequence of mean energies including the interactions inside a partition. The second moment of the Hamiltonian,

$$
\overline{H_{\alpha p}^{2}} \equiv \frac{1}{D_{\alpha p}} \operatorname{Tr}^{(\alpha p)} H^{2},
$$

includes the processes of inter-partition interactions. Due to the chaotic nature of the majority of interactions, the contribution of a partition to the many-body level density is very fast converging to a Gaussian $G_{\alpha p}(E)$ centered at $E_{\alpha p}$ of eq. (2) and having a width

$$
\sigma_{\alpha p}=\overline{H_{\alpha p}^{2}}-{\overline{H_{\alpha p}}}^{2} .
$$

Finally, the total level density is given by the superposition of those Gaussians,

$$
\rho_{\alpha}(E)=\sum_{p} D_{\alpha p} G_{\alpha p}(E) .
$$

We do not discuss here technical subtleties of this method [4]: the Gaussians $G$ have a finite range being cut at the edges, because of that they have to be renormalized; energy is counted from the ground state that has to be located correctly; in an orbital space that includes transitions between oscillator shells, one has to exclude nonphysical admixtures of spurious states related to the center-of-mass motion. There are reliable ways to handle these problems avoiding the huge diagonalization (traces are calculated directly from the matrix of $H$ ). Practically it might be convenient to use the $M$-scheme avoiding the heavy algebra of angular momentum. In the majority of cases the $M$ dependence agrees with what is frequently assumed with the use of a spin-cutoff factor. This supports the idea of random coupling of individual spins (the so-called geometric chaoticity); the exceptions are known when special values of total spin and/or isospin are excluded by the model [10]. The level density $\rho_{J}(E)$ is extracted as a difference of $\rho_{M=J}-\rho_{M=J+1}$. 
This procedure produces the level densities in various sectors essentially coinciding with the results of the full diagonalization. Figure 1 compares the shell-model solution and the moment method, the full agreement is obvious. There are small deviations close to the center which can be removed by adding the fourth moment but anyway this region of energy is outside of the physical applicability of the truncated shell model.

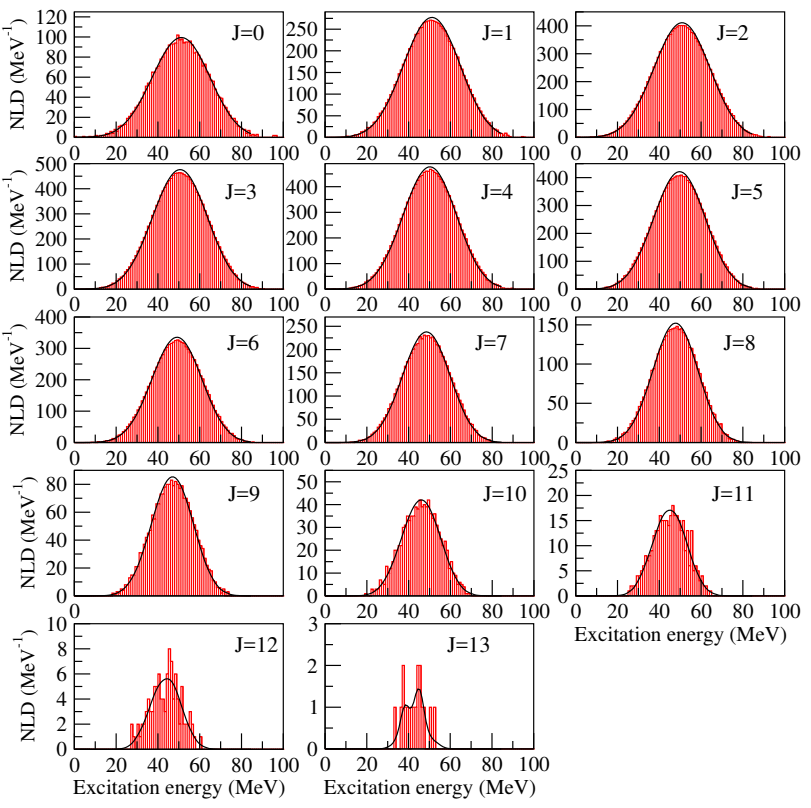

Figure 1. Level densities for various classes of states in the $s d$ shell model of ${ }^{28} \mathrm{Si}$ calculated by the full diagonalization (histograms) and by the moments method (enveloping lines).

\section{Collective enhancement}

The configuration interaction approach allows one to study the role of individual matrix elements of the shell-model Hamiltonian looking essentially inside a black box of diagonalization. It is known that even random interactions respecting the rotational symmetry reproduce some features of real spectra including the appearance of collective bands. In nuclei, the pairing interaction creates an energy gap for broken pairs. However, there could appear lowlying vibrational states. If the mean field is deformed, many rotational bands appear at low energy. In average, such collective effects increase the level density at low energy [11]. Of course, the total level number in a certain orbital space is fixed.

The shell-model studies confirm the phenomenon of collective enhancement. One way of checking this is based on the earlier results [12] showing the components of the interaction responsible for the nuclear deformation. Using random interactions and finding the sets of matrix elements corresponding to the appearance of the rotational spectrum, it was shown that the main role is played by the processes when one of the particle undergoes a transition with the change of the orbital momentum $\Delta \ell= \pm 2$ (it is understandable in the spirit of the Nilsson scheme. When the corresponding matrix elements combined into a part $V_{1}$ of the Hamiltonian are varied and the interaction produces typical rotational structures, we expect the whole level density curve to shift to low energy. The simplest exercise of this type is illustrated by Figure 2, where the shell-model Hamiltonian is presented as

$$
H=h+\lambda V_{1}+V_{2},
$$

with $h$ being a set of mean-field levels, and $V_{2}$ combining all remaining interaction parts $[13,14]$.

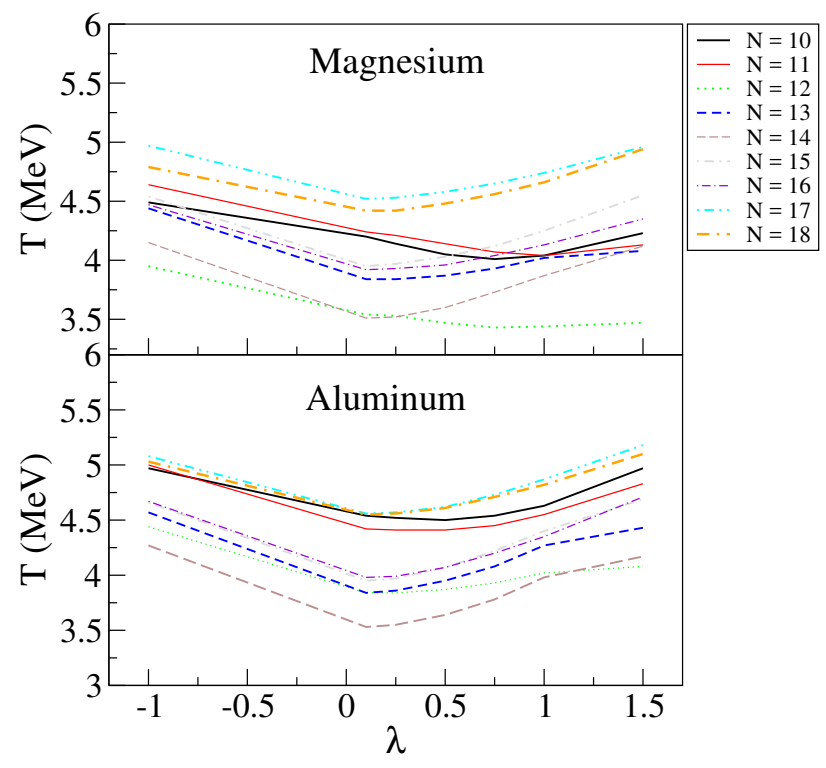

Figure 2. The collective enhancement of the low-energy level density for the interaction inducing the rotational spectrum; $N$ gives the neutron number; increase of the effective temperature $T$ corresponds to accumulation of rotational bands at low energy and slower rate of following increase of the level density to its chaotic value, see Refs. [14] and [15].

\section{Constant temperature model (CTM)}

Currently the most popular phenomenological model of the nuclear level density, suggested long ago by Ericson [16], is expressed as

$$
\rho(E)=\rho_{0} \exp (E / T)
$$

where the normalization factor is usually written as $\rho_{0}=$ $(1 / T) \exp \left(-E_{0} / T\right)$. We can call the main parameter $T$ an effective temperature. As clear from eq. (7),

$$
\frac{1}{T}=\frac{1}{\rho} \frac{d \rho}{d E}
$$

is the rate of the level density growth as a function of energy. The simple parametrization (7) is successfully used by practitioners, including the Oslo group [17].

The shell-model level density turns out to be in good agreement with this model, see Figure 3, at a significant part of the energy interval of physical applicability of a given shell-model version. The effective temperature $T$ 
systematically evolves as a function of the isotope composition, taking minimal values for $N=Z$ in even-even nuclei or in the closest isotopes for odd- $A$ or odd-odd nuclei, Figure 4 . The partial level density for the sectors with different total spins $J$ is also well described by the CTM with the slightly varying effective temperature. The complete tabulation of the level density parameters for all $s d$ nuclei can be found in Ref. [18].

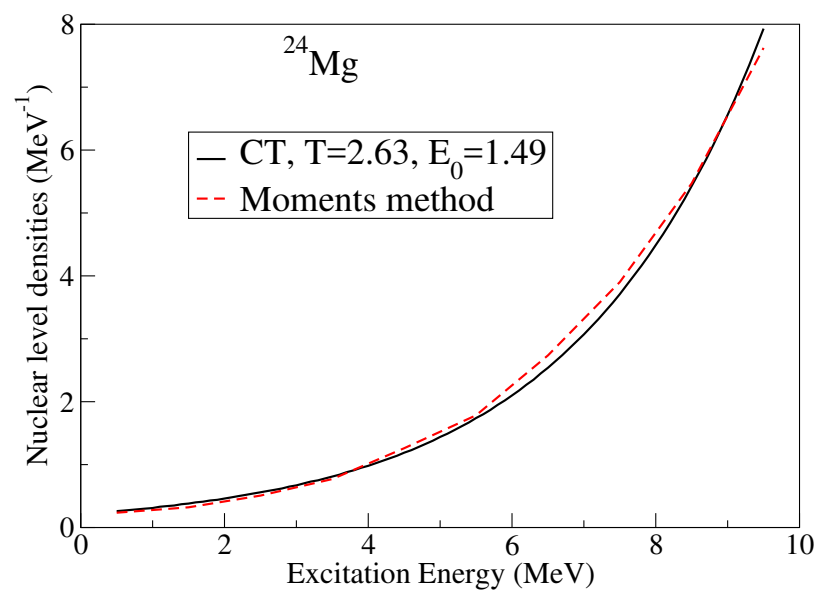

Figure 3. The shell-model level density for the $s d$-shell nucleus ${ }^{24} \mathrm{Mg}$, compared to the CTM parametrization.
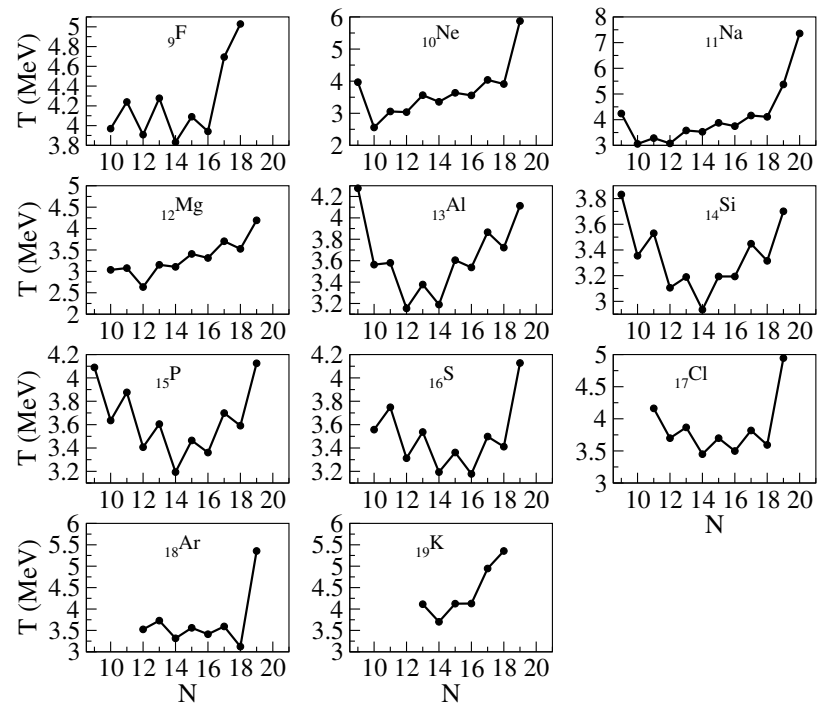

Figure 4. Systematics of the effective temperature $T$ for the $s d$ nuclei. $N$ is the neutron number.

\section{Physics of the CTM}

Traditionally, the CTM is treated [7] as a realization of a phase transition from superfluid to normal phase of nuclear matter. In accordance with that, the parameter $T$ is considered as an actual temperature kept constant while the nucleus is heated. The superfluidity is the result of pairing interactions among fermionic quasiparticles. This interpretation contradicts to the shell-model results [13].
Acting similarly to the deformation studies of Section 4, we carefully follow the evolution of the level density as a function of the pairing strength. Here, similarly to eq. (6), we single out the pairing matrix elements, $U_{1}$, and the rest, $U_{2}$,

$$
H=h+k_{1} U_{1}+k_{2} U_{2} .
$$

As shown in Figure 5, the effective temperature $T$ is practically insensitive to the changes of the pairing strength $k_{1}$, even with the transition to anti-pairing $\left(k_{1}<0\right)$.

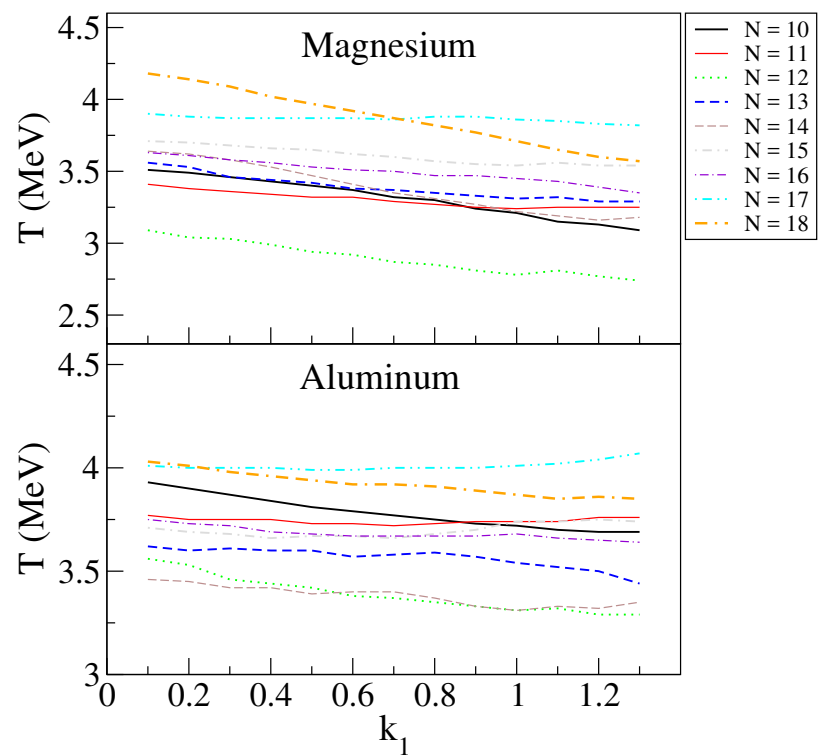

Figure 5. Effective temperature $T$ and the pairing strength $k_{1}$ for the isotopes of $\mathrm{Mg}$ and $\mathrm{Al} . N$ is the neutron number.

The effective parameter $T$ does not coincide with the thermodynamic temperature $T_{\mathrm{t}-\mathrm{d}}$ that can be defined through microcanonical entropy corresponding to the level density (7):

$$
T_{\mathrm{t}-\mathrm{d}}(E)=T\left[1-e^{-E / T}\right] .
$$

This temperature starts from zero at the ground state and grows with energy to its limiting value $T$. As known from old discussions in particle physics, where the density of resonances is exponentially growing as in the CTM, the limiting temperature signals a gradual transition to the heated chaotic stage. This transition is not directly related to pairing as seen from Figure 5. As was studied earlier [15], the nuclear pairing effects go out with energy through slow crossovers. Another significant check was done with bringing all single-particle energies to degeneracy. Then the CTM description remains valid but the effective temperature $T$ goes down. Accordingly, the rate $1 / T$ of increase of the level density grows, as expected for easily mixed degenerate mean-field orbitals. The largest rate of chaotization appearing close to the line $N=Z$ is related to the presence of all isospin values in such nuclei.

The exponential regime (7) of the level density cannot continue too far. With onset of quantum chaos, the CTM level density has to match the global Gaussian curve. Near this crossing we should have $T_{\mathrm{t}-\mathrm{d}}=T_{\text {global }}$. This is indeed the case as argued in Ref. [19]. 


\section{Role of incoherent interactions}

Many developed approaches to the nuclear level density account for the few types of interparticle interactions only. This is natural for theories based on the self-consistent mean field, even with addition of collective modes. Such a limitation is hard to avoid in Monte Carlo approaches due to their notorious sign problem. The shell-model approaches are currently the only ones working with the whole set of interactions. As shown in [10], the presence of incoherent processes (included in terms $k_{2}$ in eq. (9) is necessary in order to obtain the level density as a smooth bell-shape curve; the corresponding contributions are added in quadratures.

In the next development it could be appropriate to substitute the parts of the interaction not defined directly in physical observables by random interactions of suitable strength. Another necessary line of research is the physics of continuum and overlapping resonances.

\section{References}

[1] V. Zelevinsky, Annu. Rev. Nucl. Part. Sci. 46, 237 (1996)

[2] S. Hillaire, M. Girod, S. Goriely, and A.J. Koning, Phys. Rev. C 86, 064317 (2012)

[3] V. Zelevinsky, B.A. Brown, N. Frazier, and M. Horoi, Phys. Rep. 276, 85 (1996)

[4] R.A. Sen'kov, M. Horoi, and V. Zelevinsky, Comp. Phys. Comm. 184, 215 (2003)
[5] G.H. Lang, C.W. Johnson, S.E. Koonin, and W.E. Ormand, Phys. Rev. C 48, 1518 (1993)

[6] H. Nakada and Y. Alhassid, Phys. Rev. Lett. 79, 2939 (1997)

[7] L.G. Moretto, A.C. Larsen, F. Giakoppo, M. Guttormsen, and S. Siem, J. Phys. Conf. Series, 580, 012048 (2015)

[8] A. Gilbert and A.G.W. Cameron, Can. J. Phys. 43, 1446 (1965)

[9] S.S.M. Wong, Nuclear Statistical Spectroscopy (Oxford University Press, 1986)

[10] R. Sen'kov and V. Zelevinsky, Phys. Rev. C 93, 064304 (2016)

[11] A.V. Ignatyuk, K.K. Istekov, and G.N. Smirenkin, Sov. J. Nucl. Phys. 29, 450 (1979)

[12] M. Horoi and V. Zelevinsky, Phys. Rev. C 81, 034306 (2010)

[13] S. Karampagia and V. Zelevinsky. Phys. Rev. C 94 , 014321 (2016)

[14] S. Karampagia, A. Renzaglia, and V. Zelevinsky, Nucl. Phys. A 962, 46 (2017)

[15] M. Horoi and V. Zelevinsky, Phys. Rev. C 75, 054303 (2007)

[16] T. Ericson, Adv. Phys. 9, 425 (1960)

[17] M. Guttormsen et al., Phys. Rev. C 89, 014302 (2014)

[18] S. Karampagia, R.A. Sen'kov, and V. Zelevinsky, Atom. Data a\& Nucl. Data Tables 120, 1 (2018)

[19] V. Zelevinsky, S. Karampagia, and A. Berlaga, Phys. Lett. B, 783, 428 (2018) 